SHORT REPORT

\title{
Enhanced oxidative stress in workers with a standing occupation
}

\author{
R Flore, L Gerardino, A Santoliquido, R Pola, A Flex, C Di Campli, P Pola, P Tondi
}

Occup Environ Med 2004;61:548-550. doi: 10.1136/oem.2003.008805

Background: Several epidemiological studies have shown a statistically significant association between standing work and chronic venous insufficiency of lower limbs. This condition has been associated with an enhanced oxidative stress that, according to the literature, could represent a risk factor for cardiovascular and other systemic diseases.

Aims and Methods: To evaluate venous pressure of the lower limbs and reactive oxygen species (ROS) before and after work in 62 workers with a standing occupation (surgery room nurses) and 65 outpatient department nurses who can walk during their working time.

Results: After work, a statistically significant increase of venous pressure of the lower limbs levels was observed in both the study group and controls. Standing workers showed significantly higher mean levels of ROS after work.

$\mathrm{V}$ enous hypertension and chronic venous insufficiency (CVI) occur frequently in the working population, especially in workers with a standing profession. Several epidemiological studies have shown a statistically significant association between CVI and duration of standing work, although further data need to be collected and the role of preventive measures fully evaluated. ${ }^{12}$

Venous hypertension and CVI have been associated with haemodynamic, metabolic, and nutritional changes, such as modifications of blood flow, tissue hypoxia, and platelet and leucocytes activation. ${ }^{3}{ }^{4}$ Platelets contribute to the inflammatory response and activate the coagulation cascade. Leucocytes are able to modify the microcirculation by plugging and margination and induce cell damage by secretion of cytokines, lysosomal proteases, and reactive oxygen species (ROS). ROS are also able to initiate processes involved in atherogenesis through several important enzyme systems, including xanthine oxidase, nicotinamide adenine dinucleotide phosphate (NADPH) oxidases, and nitric oxide synthase, enhancing general cardiovascular risk. ${ }^{5}$ Oxidative stress has been also associated with other systemic diseases (cancer, degenerative disorders, etc).?

Previous work by our group has shown a local hyperproduction of ROS in the lower limbs of patients affected by varices of the long saphenous vein after one hour of upright position, while no difference was observed after one hour of recumbent position. ${ }^{8}$ Moreover we showed that the ROS hyperproduction in varicose patients can be corrected by long saphenous vein stripping. ${ }^{9}$

The aim of the present study is to evaluate whether, in healthy workers with a standing profession, a hyperproduction of ROS in the systemic circulation is detectable after work.

\section{METHODS}

We consecutively examined 98 surgery rooms nurses and 100 outpatient department nurses who work from 7 am to $2 \mathrm{pm}$ at the Gemelli University Hospital in Rome.

All subjects filled in a questionnaire about personal data, habits, family risk for venous diseases, previous pregnancies, pathological history, and habitual intake of drugs. Before work they also underwent an EchocolorDoppler examination of the lower limbs (Acuson X/P128, USA) in order to assess the presence of CVI or deep venous reflux. ${ }^{10}$

Exclusion criteria were: pregnancy, cancer, diabetes, hypertension, cardiovascular diseases (including CVI and postphlebitic syndrome), deep venous reflux, skin ulcers, recent infections, current habitual smoking, and use of antioxidant substances or oestrogens/progestins. Three males in each group were excluded because of their small number, which could confound the results.

According the above mentioned criteria, we enrolled 62 surgery room workers (group 1), who stand at the surgical table without walking for more than six hours during their shift (more than $90 \%$ of the working time), and 65 outpatient department nurses as the control group (group 2), who spend up to 3.5 hours standing during their shift (up to about $50 \%$ of the working time). Subjects gave their written informed consent and the study was approved by the Catholic University Committees.

Group 1 was composed of 62 females (mean age 45.32 (6.70) years, BMI $\left.24.46(3.12) \mathrm{kg} / \mathrm{m}^{2}\right)$; group 2 was composed of 65 females (mean age 45.65 (8.47) years, BMI 25.44 (3.99) $\mathrm{kg} / \mathrm{m}^{2}$ ).

Subjects were recommended not to wear any compression socks or stockings during their work that day. All workers were examined in a constant temperature room $\left(20^{\circ} \mathrm{C}\right)$ before and after work and underwent the following:

- Venous pressure measurement of the lower limbs: while the subject was standing we applied a sphygmomanometer tourniquet at the calf and measured the pressure at the long saphenous vein root with a Doppler $8 \mathrm{mHz}$ probe in each limb according to a standardised method. ${ }^{11}$

- Blood collection from the antecubital vein, using a 21 gauge syringe.

\section{Measurement of reactive oxygen metabolites}

Blood was centrifuged at $3000 \mathrm{rpm}(0.65 \mathrm{~g})$ for 10 minutes; serum was separated and analysed for the presence of ROS with the dROMs test (DIACRON, GR, Italy). ${ }^{8}$ Briefly, for each patient, $5 \mu \mathrm{l}$ of serum or standard were added to a solution containing $1 \mathrm{ml}$ of acetate buffer $(\mathrm{pH} 4.8)$ and $10 \mu \mathrm{l}$ of chromogen. After incubation at $37^{\circ} \mathrm{C}$ for 75 minutes, samples were analysed by spectrophotometry (Beckman

Abbreviations: BMI, body mass index; CVI, chronic venous insufficiency; ROS, reactive oxygen species 


\section{Main messages}

- On average, healthy workers with a standing occupation show an enhanced production of reactive oxygen species in the systemic circulation in comparison with ambulatory workers from the same profession.

- After work, the oxidative stress threshold is exceeded in most standing workers.

DU640) at $505 \mathrm{~nm}$ wavelength. Results were calculated using the following formula:

$$
\text { ROS }=\frac{\text { Sample absorbance }}{\text { Standard absorbance }} \times \text { Standard concentration }
$$

All measurements were performed in a blinded fashion. Data are expressed in conventional units (U); l unit corresponds to a concentration of $0.08 \mathrm{mg} / \mathrm{dl}$ hydrogen peroxide $\left(\mathrm{H}_{2} \mathrm{O}_{2}\right)$. The normal range is 200-300 U, while values higher than $300 \mathrm{U}$ indicate oxidative stress (oxidative stress threshold). ${ }^{89}$

\section{Statistics}

Data are presented as mean (SD). Comparison between groups was performed by a $t$ test and Wilcoxon test, on the basis of distribution of the data. Comparison between the changes within each group was done by a paired $t$ test.

All analyses were done by using Intercooled Stata 6.0 for Windows (Statistics/Data Analysis, Stata Corporation, College Station, Texas, USA). Statistical significance was established at $\mathrm{p}<0.05$.

\section{RESULTS}

Results are shown in table 1. Standing workers and controls were comparable for age, sex, BMI, and basal venous pressures of the lower limbs $(\mathrm{p}=\mathrm{NS})$. In the study group (group 1), venous pressure of the right leg increased from 56.05 (21.64) to 84.60 (17.54) $\mathrm{mm} \mathrm{Hg}$ after work; venous pressure of the left leg also increased from 55.64 (19.97) to 83.39 (17.57) $\mathrm{mm} \mathrm{Hg}$ after work. In the controls (group 2), venous pressure of the right leg increased from 56.23 (18.14) to 76.54 (17.30) $\mathrm{mm} \mathrm{Hg}$ after work; venous pressure of the left leg also increased from 56.33 (16.70) to 75.39 (16.70) $\mathrm{mm} \mathrm{Hg}$ after work. In both study group and controls, the increase of venous pressure after work was statistically significant $(\mathrm{p}<0.0001)$. However, the venous pressures after work was significantly higher in the study group

\section{Policy implications}

- Detection of workers at risk for oxidative stress is possible by measurement of reactive oxygen species before and after work.

- Healthy workers could also be at risk for enhanced oxidative stress because of a standing profession.

- Further research is needed to evaluate whether common prevention measures (such as compression stockings, rest breaks, mini breaks, chances for ambulation while working) are able to correct the oxidative stress related to standing occupations.

both in the right and in the left leg $(p=0.01)$. Similarly the percentage change from pre-work level of venous pressure after work was significantly higher than that observed in controls in both the left $(p<0.01)$ and right $(\mathrm{p}<0.05)$ lower limbs. In healthy subjects, venous pressure taken on the right and the left legs of the same individual is correlated and so we expected to obtain similar results for both legs.

In standing workers, ROS before work was 285.10 (69.82) $\mathrm{U}$; it increased to $338.51(82.76) \mathrm{U}(\mathrm{p}<0.0001)$ at the end of work, above the oxidative stress threshold (300 U). In controls we observed a non-statistically significant increase of ROS from a basal value of 253.94 (66.70) to 263.09 (63.53) after work.

The comparison between groups showed higher basal values of ROS in standing workers (285.10 (69.82) U v 253.94 (66.70) U; p < 0.05) and after work (338.51 (82.76) v 263.09 (63.53); $<<0.0001)$. Moreover, the percentage changes from the pre-work level of ROS in the two groups were statistically significant $(\mathrm{p}<0.05)$.

We also evaluated the number of subject with ROS values over the oxidative stress threshold (300 U) in each group before and after work. In standing workers 21 subjects (34\%) exceeded the oxidative stress threshold before work and 38 (61\%) after work; in controls $13(20 \%)$ exceeded 300 U before work and $16(24 \%)$ after work. The comparison between groups was statistically significant only after work (odds ratio $4.85 ; 95 \%$ CI 2.3 to $10.3 ; \mathrm{p}<0.0001)$.

\section{DISCUSSION}

It is widely known that people with standing professions present with venous hypertension of the lower limbs, which activates local mechanisms responsible for the onset and the

Table 1 Mean (SD) with 95\% Cl of venous pressure (VP) in both limbs and reactive oxygen metabolites (ROS) before and after work in the two groups; the mean percentage changes between pre-work and after-work level in the two groups are also shown

\begin{tabular}{|c|c|c|c|c|c|}
\hline & \multicolumn{2}{|l|}{ Group 1} & \multicolumn{2}{|l|}{ Group 2} & \multirow[b]{2}{*}{ p } \\
\hline & Mean (SD) & $95 \% \mathrm{Cl}$ & Mean (SD) & $95 \% \mathrm{Cl}$ & \\
\hline \multirow[t]{2}{*}{ VP before $(\mathrm{mm} \mathrm{Hg})$} & Left: 55.64 (19.97) & Left: 50.57 to 60.71 & Left: 56.33 (16.70) & Left: 52.16 to 60.50 & Left: NS \\
\hline & Right: 56.05 (21.64) & Right: 50.55 to 61.54 & Right: 56.23 (18.14) & Right: 51.74 to 60.72 & Right: NS \\
\hline \multirow[t]{2}{*}{ VP after (mm Hg) } & Left: 83.39 (17.57) & Left: 78.92 to 87.85 & Left: 75.39 (16.70) & Left: 71.15 to 79.63 & Left: 0.01 \\
\hline & Right: 84.60 (17.54) & Right: 80.14 to 89.05 & Right: 76.54 (17.30) & Right: 72.25 to 80.82 & Right: 0.01 \\
\hline \multirow[t]{2}{*}{$p$ (before $v$ after) } & Left: $<0.0001$ & & Left: $<0.0001$ & & \\
\hline & Right: $<0.0001$ & & Right: $<0.0001$ & & \\
\hline \multirow[t]{2}{*}{ VP $\Delta \%$} & Left: 70.28 (79.22) & Left: 50.16 to 90.40 & Left: 40.95 (40.21) & Left: 30.90 to 50.99 & Left: $<0.01$ \\
\hline & Right: 77.06 (95.67) & Right: 52.77 to 101.36 & Right: 45.71 (34.08) & Right: 34.08 to 57.33 & Right: $<0.05$ \\
\hline ROS (U) before & $285.10(69.82)$ & 266.30 to 301.73 & $253.94(66.70)$ & 236.49 to 269.50 & $<0.05$ \\
\hline ROS (U) after & $338.51(82.76)$ & 315.60 to 357.88 & $263.09(63.53)$ & 246.25 to 277.78 & $<0.0001$ \\
\hline P (before $v$ after) & $<0.0001$ & & NS & & \\
\hline ROS $\Delta \%$ & $20.90(25.33)$ & 14.47 to 27.34 & $9.10(38.17)$ & -0.36 to 18.56 & $<0.05$ \\
\hline
\end{tabular}


evolution of CVI. ${ }^{1-3}$ Among the mediators of vessel wall damage, ROS play an important role, acting through two main mechanisms. The first is the oxidation of cell membrane components, followed by endothelium damage, which eventually leads to increased vascular permeability. The second mechanism is the chemotaxis and activation of leucocytes and platelets. As these mechanisms work together, cell damage is amplified and venous stasis is increased..$^{5}$

According to our previous findings, after venous stasis induced by 60 minutes of upright position, ROS production is significantly higher in patients with varicose veins than controls, ${ }^{8}$ and this alteration can be corrected by long saphenous vein stripping. ${ }^{9}$ In the present study we observed that most healthy workers (not affected by CVI) with a standing profession have a hyperproduction of ROS (over the oxidative stress threshold) after work. Furthermore, before work, ROS levels are on average significantly higher in standing workers. Conversely, we found no significant increase of mean ROS levels in workers who can walk during work time. However, since not all standing workers show ROS levels above the oxidative stress threshold, and conversely a few controls have results above the threshold, other protective and damaging factors apart from the standing profession could be present.

Several studies have shown that oxidative stress is an important risk factor for cardiovascular and other systemic diseases. Our data showing an enhanced oxidative stress in standing workers may represent an important step forward in the evaluation of professional risks, although further studies are needed to assess whether ROS production could be a risk factor for systemic diseases. It would be advisable to avoid the hyperproduction of ROS in people with standing professions, by means of suitable preventive measures (compressive stockings and, if possible, rest breaks, mini breaks, or chances for ambulation while working, etc). We are currently investigating the prevention of this phenomenon.

\section{Authors' affiliations}

R Flore, L Gerardino, A Santoliquido, A Flex, C Di Campli, P Pola, Department of Medicine, A. Gemelli University Hospital, Catholic University of the Sacred Heart, Rome, Italy

P Tondi, Division of Vascular Medicine, A. Gemelli University Hospital, Catholic University of the Sacred Heart, Rome, Italy

R Pola, Laboratory of Vascular Biology and Genetics, A. Gemelli

University Hospital, Catholic University of the Sacred Heart, Rome, Italy

The research was funded by an unrestricted grant from ISPESL (National Institute for Occupational Safety and Prevention, Italy)

Correspondence to: Dr R Flore, Department of Medicine, Catholic University of the Sacred Heart, A. Gemelli University Hospital, Largo A. Gemelli, 8-00165 Rome, Italy; angiologia@rm.unicatt.it

Accepted 4 August 2003

\section{REFERENCES}

1 Krijnen RM, de Boer EM, Ader HJ, et al. Venous insufficiency in male workers with a standing profession. Part 1: Epidemiology. Dermatology 1997; 194:111-20.

2 Kontosic I, Vukelic M, Drescik I, et al. Work conditions as risk factors for varicose veins of the lower extremities in certain professions of the working population of Rijeka. Acta Med Okayama 2000;54:33-8.

3 Smith PD. Neutrophil activation and mediators of inflammation of chronic venous insufficiency. J Vasc Res 1999;36(suppl 1):24.

4 Cordts PR, Kaminski MV, Raju S, et al. Could gut-liver function derangements cause chronic venous insufficiency? Vasc Surg 2001;35:107-14.

5 Harrison D, Griendling KK, Landmesser U, et al. Role of oxidative stress in atherosclerosis. Am J Cardiol 2003;91(3A):7A-11A.

6 Fukai T, Folz RJ, Landmesser U, et al. Extracellular superoxide dismutase and cardiovascular disease. Cardiovasc Res 2002;55:239-49.

7 Migliore L, Coppede F. Genetic and environmental factors in cancer and neurodegenerative diseases. Mutat Res 2002;512:135-53.

8 Pola P, Dal Lago A, Flore R, et al. Hyperproduction of oxygen free radicals in non complicated varicose disease of the lower limbs: preliminary data. J Vasc Invest 1998:4:25-30.

9 Flore R, Santoliquido A, Dal Lago A, et al. Long saphenous vein stripping reduces local level of reactive oxygen metabolites in patients with varicose disease of the lower limbs. World J Surg 2003;27:473-5.

10 Welch HJ, Young CM, Semegran AB, et al. Duplex assessment of venous reflux and chronic venous insufficiency: the significance of deep venous reflux. J Vasc Surg 1996;24:755-62.

11 Bartolo M, Antignani PL, Di Folca, et al. Mesure de la pression veineuse avec le Doppler: standardisation de la méthode. Phlébologie 1984;37:97-101. 